\title{
Adnexal torsion in a heterotopic pregnancy: a rare clinical entity with diagnostic dilemma
}

\section{Kirtirekha Mohapatra*, Subhasri Mishra, Balaram Sahoo, Basudev Marandi, Rishika Sen, Sagarika N.}

Department of Obstetrics \& Gynaecology, SCB Medical College, Cuttack, Odisha, India

\author{
Received: 17 October 2015 \\ Revised: 23 October 2015 \\ Accepted: 14 December 2015 \\ *Correspondence: \\ Dr. Kirtirekha Mohapatra, \\ E-mail: kirtirekhamohapatra@gmail.com
}

Copyright: ( $)$ the author(s), publisher and licensee Medip Academy. This is an open-access article distributed under the terms of the Creative Commons Attribution Non-Commercial License, which permits unrestricted non-commercial use, distribution, and reproduction in any medium, provided the original work is properly cited.

\begin{abstract}
Adnexal torsion is an uncommon cause of surgical emergency during pregnancy. Its incidence is 1 in 5000 pregnancies, occurring more frequently in the first trimester after ovarian hyper stimulation. Heterotopic pregnancy is also a rare entity and cause pain abdomen but without the classical symptom of bleeding per vaginum. Heterotopic pregnancy with adnexal torsion is extremely rare but increases the case fatality rate. Hence the differential diagnosis of pain abdomen during early pregnancy both may be considered and it should not be forgotten that adnexal torsion may occur in absence of ovarian cyst. We report a case of adnexal torsion that was successfully managed by laparoscopy followed by laparotomy during pregnancy and diagnosed as a heterotopic pregnancy after getting the histopathological report. A 24-year-old G2P1 admitted to LR of SCBMCH at 4 weeks of GA with severe left abdominal pain and nausea for one day. Her vitals were stable. There was tenderness on the left iliac fossa with cervical motion tenderness and left POD fullness and tenderness, TAS visualized a foetus in utero with GA of 7 weeks and a hyperechoic mass of $6 \times 6 \mathrm{~cm}$ on left side. Laparotomy done as the mass was gangrenous, enlarged and haemorhagic. Total salpingo oophorectomy of left side was done. Postoperative period was uneventful and managed with progesterone to continue the pregnancy. Histopathology report confirmed heterotopic pregnancy. This case suggests that clinicians should suspect both heterotopic pregnancy and adnexal torsion while evaluating pregnant patients with pain abdomen.
\end{abstract}

Keywords: Adnexal torsion, Heterotopic pregnancy, Hyperechogenic mass

\section{INTRODUCTION}

Adnexal torsion is an uncommon cause of surgical emergency during pregnancy. It accounts for $2.7 \%$ of all gynecological emergencies. ${ }^{1}$ Its incidence is 1 in 5000 pregnancies, occurring more frequently in the first trimester after ovarian hyper stimulation. ${ }^{2}$ Increased frequency during pregnancy is due to greater laxity of the tissues adjoining the ovaries and tubes as well as to enlargement of the ovary in early pregnancy secondary to corpus luteum cyst. Traditionally adnexal torsion presenting as acute abdomen is managed by laparotomy. Adnexal torsion is the total or partial rotation of the adnexa around its vascular axis or pedicle. The exact aetiology is obscure. Complete torsion causes venous and lymphatic blockade leading to stasis and venous congestion, haemorrhage and necrosis. Patient usually presents with acute abdomen and pelvic examination may reveal a tender cystic mass separate from the uterus. The risk of adnexal torsion rises by 5-fold during pregnancy and associated with ovarian stimulation for IVF, ectopic pregnancy in fallopian tube or ovarian masses. ${ }^{2}$ As this is rare in pregnancy, high index of suspicion is required in the first trimester. Diagnosis on pure clinical grounds is often difficult. The exact role of imaging techniques is debated. Adnexal torsion is a rare cause of acute 
abdominal pain during pregnancy. It is uncommon for a normal-sized ovary to undergo torsion. Adnexal torsion is more common on the right side, perhaps because the sigmoid colon leaves a limited space for left adnexal mobility and it is very rarely bilateral.

Heterotopic pregnancy is the coexistence of intrauterine and extra uterine pregnancies. It has been traditionally regarded as a very rare event with an estimated incidence of 1 in 30,000 pregnancies. $^{3}$ It rarely occurs in spontaneous conception cycles. It is definitely a fatal condition that might even lead to maternal morbidity and even mortality if there is a delay in intervention assuming it to be an intrauterine pregnancy (IUP). IUP diagnosed with ease in this case will enumerate how the source of haemoperitoneum to be of tubal origin but because of the rarity of the incidence of heterotopic pregnancy it may be missed.

\section{CASE REPORT}

A 24 year-old G 2 P 1 presented to the labour room of SCB medical college and Hospital at 4 weeks 4 days of gestation based on the last menstrual period with a new onset of severe left abdominal pain not responding to analgesia and nausea for one day. She described the pain as sharp non-radiating type of pain in the left iliac fossa with a sudden onset, with no relieving factors. She had done a urine pregnancy test at home which was positive. Then she came to SCB MCH to terminate the pregnancy. She gave no history of vaginal bleeding or discharge. There was no history of diarrhoea, constipation, fever, urinary complaints or any recent illness. She did not present any risk factors for adnexal torsion such as ovarian hyper stimulation, ovarian cysts, and previous history of torsion. No history of recent sexual intercourse or sudden movement. Her first pregnancy was a term male child delivered vaginally in hospital nine months back.

On general examination, patient was conscious, with pulse $100 / \mathrm{min}$, temperature $38^{\circ} \mathrm{C}$, blood pressure $100 / 74$ $\mathrm{mmHg}$, cardiovascular and respiratory systems were normal. Abdominal examination revealed tenderness on the left in left inguinal region. There were no signs of peritoneal irritation, no engorged veins, no guarding or rigidity. Umbilicus was central and inverted. The pelvic examination revealed cervical motion tenderness as well as left adnexal fullness and tenderness, with no vaginal discharge. All her blood and urine investigations were within normal limits. Transabdominal sonography visualized a foetus in utero with a gestational age of 7weeks 2 days. Adjacent to the uterus on the left side, there was a hyperechogenic mass measured $6 \times 6 \times 6 \mathrm{~cm}$ with well-defined contours. Both ovaries were visible and there was evidence of free intraperitoneal fluid collection. Doppler studies showed decreased blood flow in left adnexa. Images were interpreted by the radiologist to represent a left adnexal torsion. The patient was counseled concerning the risk of abortion and the possibility of adnexal torsion, and informed consent for abdominal exploration with the possible need for salpingo-oophorectomy was obtained.

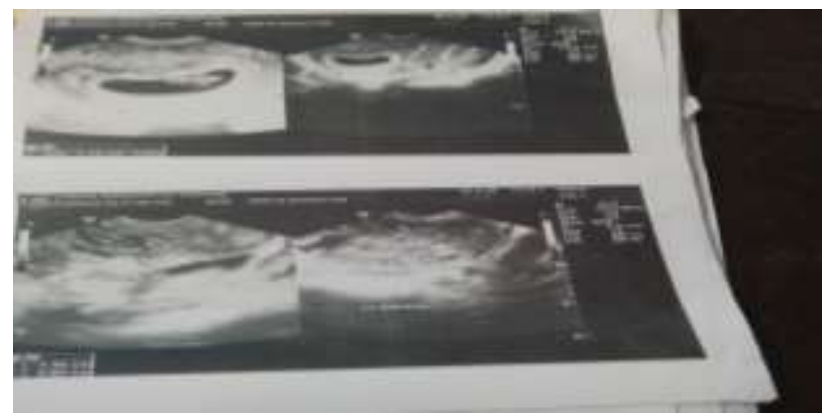

Figure 1: USG shows IU pregnancy with left side adnexa.

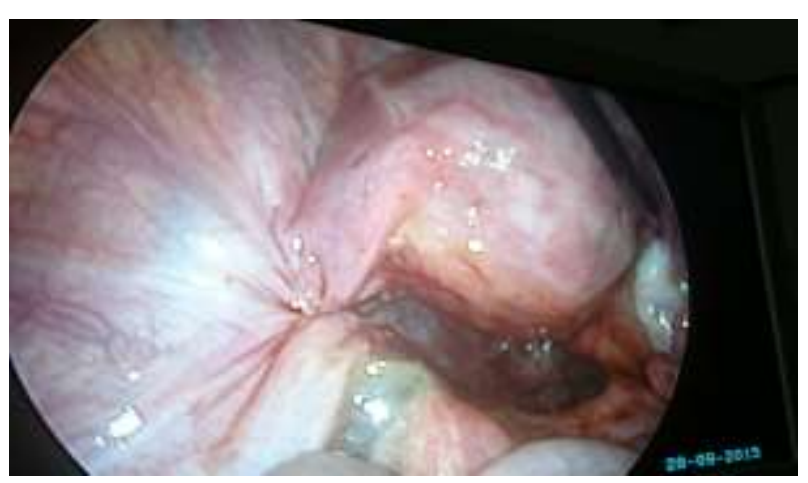

Figure 2: Left adnexal torsion with gangrenous mass Seen in laparoscopic view.

She was initially managed conservatively with antibiotics IV fluid, analgesics, inj HCG, and progesterone in view of continuing the pregnancy. She still complained of dull aching pain in her lower abdomen which persisted in spite of the medications. Hence she was planned for a laparoscopic evaluation of the adnexal mass and planned for surgery. On Laparoscopy uterus was found to be bulky and normal in contour. Right side tube and ovary healthy, on left side the entire tubo ovarian complex was twisted and adhered to the POD and omentum and seemed blackish in colour owing to the ongoing necrotic activity, hence decision for laparotomy was taken. Abdomen was opened by Pfannenstiel incision and a black gangrenous enlarged, haemorrhagic, left adnexal mass burying the tube and ovary which was twisted two rounds and adherent to the omentum was found. The adhesions were removed by blunt dissection and rerotation was done carefully, but no improvement in colour or decrease in edema of the adnexa was seen. The entire mass was dissected out by clamping, cutting and ligating the stump i.e. total salpingo oophorectomy of left side was performed. Right tube and ovary were healthy. Proper hemostasis was maintained. No free intraperitoneal fluid collection was found. Adnexa was cut open, fallopian tube showed blood clots and some trophoblastic tissue like structures. The postoperative course was uneventful, and an intact intrauterine 
pregnancy was confirmed 2 days postoperative by USG. Our case was diagnosed as a case of heterotopic pregnancy after getting the histopathological report.

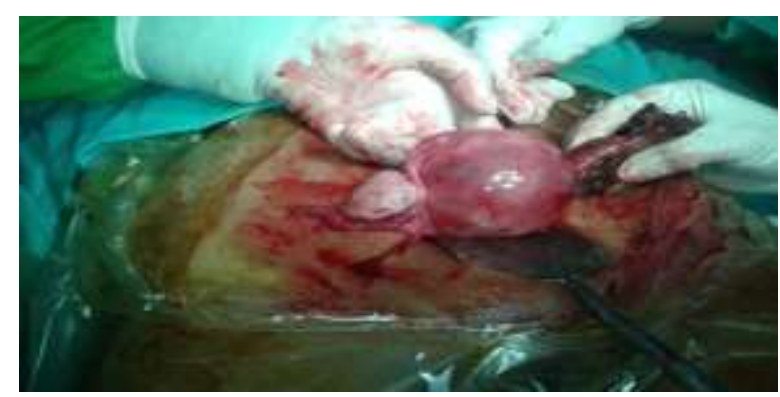

Figure 3: Left adnexal torsion with gangrenous mass seen in laparotomy.

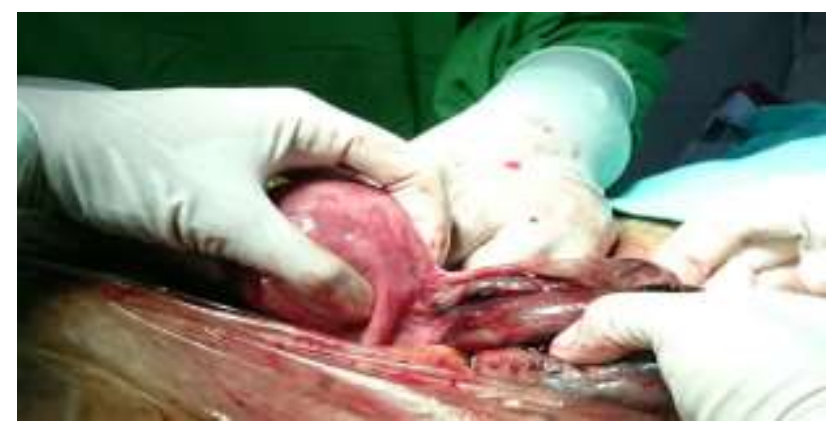

Figure 4: Left adnexal torsion with gangrenous mass seen laparotomy.

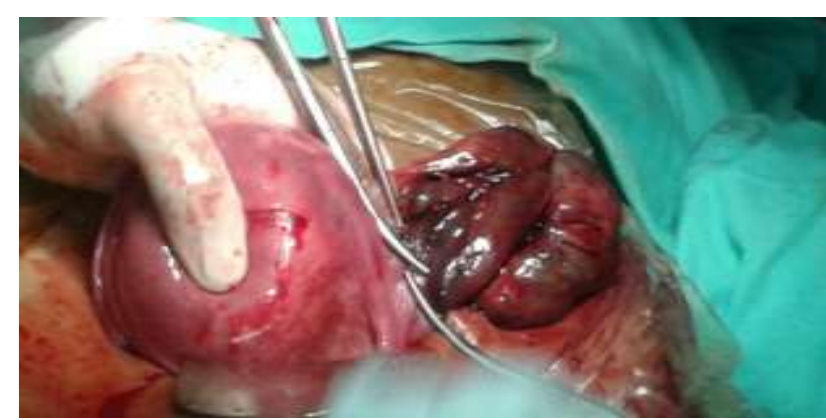

Figure 5: Clamps given for left side salpingoophorectomy.

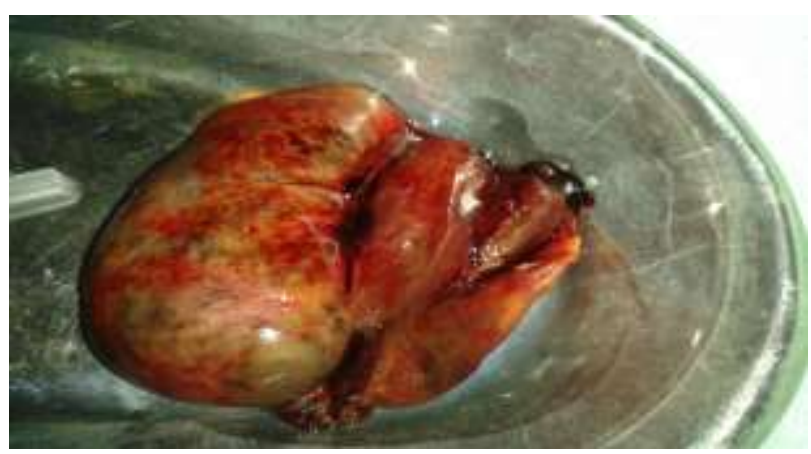

Figure 6: Specimen of left adenexa after Salpingoophorectomy.

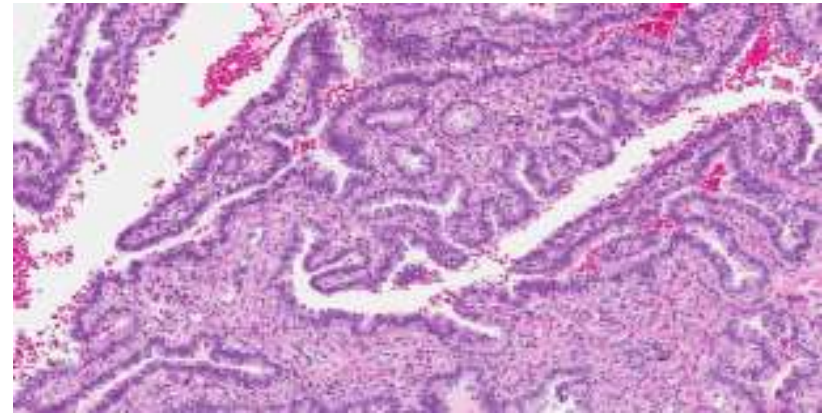

Figure 7: Microscopic picture shows trophoblastic villi suggesting that an ectopic pregnancy.

\section{DISCUSSION}

The heterotopic pregnancy is a combination of an intrauterine and extra-uterine pregnancy at the same time. The spontaneous heterotopic pregnancy is a rare illness with an estimated frequency below one per 20,000 and one per $30,000 .{ }^{3}$ The first case was reported in France by Duverney in 1708 during an autopsy. ${ }^{4}$ Although spontaneous simultaneous intrauterine and ectopic pregnancy was an extremely rare event in the past, it is increasingly being diagnosed since the rate of assisted reproductive technique increased. The preoperative diagnosis of a heterotopic pregnancy remains a major challenge for modern reproductive medicine. Although signs and symptoms such as abdominal pain, adnexal mass, peritoneal irritation, vaginal bleeding, adnexal tenderness and enlarged uterus have been reported to be predictive of a heterotopic pregnancy, they are nonspecific and may be confused with other normal or abnormal pregnancy manifestations. Furthermore, if assisted reproductive technique is not involved, the index of suspicion of multiple or heterotopic pregnancy is usually very low. Heterotopic pregnancy is a rare condition and most patients present in the emergency department with symptoms of a rupture of ectopic component. ${ }^{5}$ Thus, a preoperative diagnosis of heterotopic pregnancy is still a challenge. The advent of ultrasound for pregnancy positive patient has not changed diagnostic ability over a period of time. In a review of the literature of all cases of heterotopic pregnancy from 1971-1993, out of 112 cases, 46 were diagnosed by ultrasound while 66 were diagnosed at laparoscopy or laparotomy. ${ }^{6}$ Transvaginal ultrasound and assessment of the whole pelvis, even in the presence of intrauterine, can be an important aid in the diagnosis of heterotopic pregnancy. Serial $\beta$-hCG levels are not of much significance and diagnosis of heterotopic pregnancy as subnormal hormone production by an ectopic pregnancy may be masked by the higher placental production from the intrauterine pregnancy. ${ }^{7}$ Culdocentesis is an important aid in diagnosis when hemoperitoneum is presented as echogenic cul-de-sac fluid collection is more important than anechoic fluid because it indicates the presence of peritoneal hemorrhage. Management of heterotopic pregnancy still remains controversial. The standard treatment for ectopic pregnancy is surgery by laparoscopy 
or laparotomy depending upon the condition of the patient. After diagnosis, the ectopic component is usually treated surgically, whereas the intrauterine pregnancy is expected to develop normally. The main aim of the surgery should be the preservation of the intrauterine pregnancy with minimal manipulation of the uterus. ${ }^{8}$ Many cases are treated by surgery via laparoscopy or laparotomy, including salpingotomy or salpingectomy. The choice between conservative or radical treatments may be difficult. However, a recent review demonstrated no difference in rates of intrauterine pregnancies after conservative or radical surgery for tubal ectopic pregnancy. ${ }^{9}$ It seems that, particularly in patient with an intact contralateral tube, fertility results after salpingectomy are comparable to those observed after salpingotomy. ${ }^{10}$ Moreover, radical treatment is easier, thus reducing the risk of complication observed at salpingotomy. Laparotomy has been used widely until recently. Nowadays, laparoscopy is preferred treatment for heterotopic pregnancy and laparoscopy is the appropriate modality both for diagnosis and treatment of heterotopic pregnancy. ${ }^{11}$ However, laparotomy may be the preferable surgical modality in cases with serious intra-abdominal bleeding or in patients with hemorrhagic shock. $^{12}$ In conclusion, heterotopic pregnancy should be thought in differential diagnosis of an acute abdomen. It is important to remember that the detection of an intrauterine pregnancy does not exclude the existence of an accompanying ectopic pregnancy. In the case of heterotopic pregnancy, salpingectomy should be considered when the contralateral fallopian tube is healthy as this treatment does not preclude future fertility.

Adnexal torsion during pregnancy is a rare condition, more common in the first trimester. The symptoms are nonspecific, and can be confused with other acute abdominal conditions such as appendicitis, ureteral or renal colic, cholecystitis and bowel obstruction. The presence on ultrasound of an ovarian mass should raise the suspicion for adnexal torsion. Ideal Management of adnexal torsion in pregnancy is the laparoscopic approach combined with simple detorsion if diagnosed early, laparotomy and salpingo-oophorectomy may sometimes be necessary . Torsion of the adnexa has traditionally been treated by surgical excision of the affected structure without untwisting, because of the fear of emboli departing from thrombosed ovarian veins. Several recent reports have described successful conservative management with untwisting of the twisted adnexa. As the trend towards conservative management progresses, the incidence of recurrent adnexal torsion may increase. Torsion of adnexa is a well-established clinical entity. It is estimated that $12 \%-18 \%$ of the adnexal torsion occur during pregnancy. ${ }^{13}$ The incidence is highest during the first trimester of pregnancy, the condition being rare during second and exceptional during third trimester. The risk of ovarian torsion associated with OHSS also seems to increase if the patients do subsequently conceive. It is because of the continuous exposure of a hyperstimulated ovary with endogenous hCG resulting in an increase in ovarian size. Torsion in pregnancy presents a diagnostic challenge. The accuracy of preoperative diagnosis during pregnancy is only $70 \% .{ }^{13,14}$ Cases of adnexal torsion have been reported in association with ovarian hyperstimulation syndrome. ${ }^{14,15}$ Although the classic presentation of adnexal torsion is the acute onset of abdominal pain, an adnexal mass and clinical evidence of peritonitis, this triad is usually not present in most of the patients later found to have adnexal torsion. The presenting findings in most patients were nonspecific and unimpressive, inviting a good number of differential diagnosis, including appendicitis, pelvic inflammatory disease, hemorrhage into the cyst, ectopic/heterotopic pregnancy. Few of these conditions are not treated surgically contributing to the delay in surgical intervention. ${ }^{16}$ Early diagnosis and prompt treatment is crucial in these cases to avoid these young infertile women losing their ovaries. The use of color Doppler sonography has proved useful for such a diagnosis, the main sign being the absence of intraparenchymal ovarian blood flow. ${ }^{17}$ In young and infertile patients a loss of an ovary may be tragic. There are reports of unwinding the adnexa regardless of whether it is ischemic or not. ${ }^{14}$ This treatment has been encouraging. Diagnosis of adnexal torsion is hardly possible by noting non-specific symptoms common in pregnancy. Early diagnosis is essential as it makes a conservative approach possible. When diagnosis is made earlier, simple detorsion is possible with good functional results. Although the use of colour Doppler sonography, with the main sign of the absence of intraparenchymal ovarian blood flow indicative of adnexal torsion, seems to be a promising diagnostic tool in establishing the diagnosis, a decreased blood flow, which could have been the result of incomplete torsion, should not rule out the suspicion of adnexal torsion. Nowadays, MRI appears to be a potential alternative, as it can demonstrate signs of hemorrhagic infarction.

There are reports of unwinding the adnexa regardless of whether it is ischemic or not. Intravenous fluorescein injection followed by observation of adnexa by UV rays may confirm viability of adnexa. This treatment has been encouraging. At the present time laparoscopy is the most specific diagnostic tool for evaluating torsion recently, laparoscopic surgery during advanced pregnancy has been reported to be feasible and safe. However, it needs both skilled personnel with a wide experience in operative gyneacological laparoscopy and also sophisticated armamentorium. Laparoscopic adnexal detorsion, not adnexectomy is the procedure of choice unless the tissue is gangrenous as it was in this case. Laparoscopy during pregnancy is associated with a significantly lower prevalence of postoperative complications than laparotomy. It is not associated with higher rates of abortion, preterm deliveries, IUGR or fetal anomalies in comparison with laparotomy. ${ }^{18}$ Untwisting the adnexa to provide a satisfactory recovery, and also aspiration of ovarian cysts, are recommended as the first interventional alternatives. 
In our case, because of the large gangrenous mass with adhesion seen during laparoscopy, we performed a laparotomy.

\section{CONCLUSION}

An early diagnosis might have helped to conserve patient's ovary. Though it is an extremely rare problem in pregnancy, adnexal torsion should be taken into consideration in the differential diagnosis of abdominal pain and it should not be forgotten that adnexal torsion may occur even in the absence of ovarian cysts. In this case increased weight to initiate torsion might be provided by enlarged tube of tubal pregnancy. This case suggests that the clinician should maintain a reasonable index of suspicion while evaluating a patient with pain abdomen and amenorrhoea even in face of documented intrauterine pregnancy. An early and prompt intervention at the very first sight of heterotopic pregnancy is a must to salvage the intrauterine pregnancy and to avoid missing this potentially life-threatening condition that can lead to loss of intrauterine pregnancy, maternal morbidity and mortality.

Informed consent was taken from the patient.

\section{Funding: No funding sources}

Conflict of interest: The authors declare that they have no conflict of interest

Ethical approval: Not required

\section{REFERENCES}

1. Kolluru V, Gurumurthy R, Vellanki V, Gururaj D. Torsion of ovarian cyst during pregnancy: a case report. Cases Journal. 2009;2:9405.

2. Smorgick N, Maymon R, Mendelovic S, Herman A, Pansky M. Torsion of normal adnexa in postmenarcheal women: can ultrasound indicate an ischemic process? Ultrasound Obstet Gynecol. 2008;31(3):338-41.

3. Harris J, Finberg MD. Ultrasonography In Obstetrics and Gynaecology: Ultrasound evaluations in multiple gestations. $3^{\text {rd }}$ ed. W B Saunders company, Harcourt Brace and company. 1997:102-28.

4. Cholkeri-Singh A, LaBarge A. Spontaneous heterotopic triplets: a case report. Fertil Steril. 2007;88(4):968.e5-7.

5. Jeong HC, Park IH, Yoon YS, Lee NW, Kim HJ, $\operatorname{Kim} \mathrm{T}$ et al. Heterotopic triplet pregnancy with bilateral tubal and intrauterine pregnancy after spontaneous conception. Eur J Obstet Gynecol Reprod Biol. 2009;142(2):161-2.

6. Tandon R, Goel P, Saha PK, Devi L. Spontaneous heterotopicpregnancy with tubal rupture: a case report and review of the literature. J Med Case Reports . 2009;3:8153.

7. Umranikar S, Umranikar A, Rafi J, Bawden P, O'Sullivan B ,Moors A. Acute presentation of a heterotopic pregnancy following spontaneous conception: a case report. Cases J. 2009;2:9369.

8. Clayton HB, Schieve LA, Peterson HB, Jamieson DJ, Reynolds MA, Wright VC. A comparison of heterotopic and intrauterine only pregnancy outcomes after assisted reproductive technologies in the United States from 1999 to 2002. Fertil Steril. 2007;87(2):303-9.

9. Aust T, O’Neill A, Cario G. Purse-string suture technique to enable laparoscopic management of the interstitial gestation of a heterotopic pregnancy. Fertil Steril . 2011;95(1):261-3.

10. Jan F, Naikoo GM, Rather MH, Sheikh TA, Rather YH. Ruptured heterotopic pregnancy: a rare cause for hemoperitoneum; report of three cases from kashmir, India. Indian J Surg. 2010;72(5):404-6.

11. Simsek T, Dogan A, Simsek M, Pestereli E. Heterotopic triplet pregnancy (twin tubal) in a natural cycle with tubal rupture: case report and review of the literature. J Obstet Gynaecol Res. 2008;34(4 pt 2):759-62.

12. Lialios GA, Kallitsaris A, Kabisios T, Messinis IE. Ruptured heterotopic interstitial pregnancy: rare case of acute abdomen in a Jehovah's Witness patient. Fertil Steril. 2008;90(4):1200.e15-7.

13. Hibbard LT. Adnexal torsion. Am J Obstet Gynecol. 1985;152(4):456-61.

14. Mashiach S, Bider D, Moran O, Goldenberg M, BenRafael Z. Adnexal torsion of hyper stimulated ovaries in pregnancies after gonadotropin therapy. Fertil Steril .1990;53(1):76-80.

15. Chew S, Ng SC. Laparoscopic treatment of a twisted hyper stimulated ovary after IVF. Singapore Med J. 2001;42(5):228-9.

16. Bayer AI, Wiskind AK. Adnexal torsion: can the adnexa be saved? Am J Obstet Gynecol. 1994;171(6);1506-10.

17. Bassil S, Steinhart U, Donnez J. Successful laparoscopic management of adnexal torsion during week 25 of a twin pregnancy. Hum Reprod. 1999;14(3):885-7.

18. Oelsner G, Stockheim D, Soriano D, Goldenberg M, Seidman DS, Cohen SB et al. Pregnancy outcome after laparoscopy or laparotomy in pregnancy. J Am Assoc Gynecol Laparosc. 2003;10(2):200-4.

Cite this article as: Mohapatra K, Mishra S, Sahoo B, Marandi B, Sen R, Sagarika N. Adnexal torsion in a heterotopic pregnancy: a rare clinical entity with diagnostic dilemma. Int J Reprod Contracept Obstet Gynecol 2016;5:232-6. 